\title{
QUALITY ASSESSMENT IN SCRUM SOFTWARE DEVELOPMENT THROUGH METRICS: A CONCEPTUALIZATION USING CONCEPT MAPS
}

\author{
Sirine Zaouali ${ }^{1}$ and Sonia Ayachi Ghannouchi ${ }^{2}$ \\ ${ }^{1}$ Higher Institute of Computer Science and Communication Techniques, University of Sousse \\ 12 Beach Route, Sousse, Tunisia \\ ${ }^{2}$ High Institute on Management of Soussse, University of Sousse \\ Road of Abdlaaziz il Behi. Bp 763. 4000, Sousse, Tunisia
}

\begin{abstract}
In Scrum software development projects, plenty of quality metrics may be measured and used for quality assessment. Selecting the required metrics thus becomes a critical step. Scrum projects are fundamentally conducted around three elements: team, artifacts and events. These elements can be helpful for guiding the involved stakeholders in selecting the most pertinent ones. Hence, in this paper we propose concept maps that promote quality assessment through a classification of Scrum quality metrics based on the three aforementioned elements. These Concept Maps will guarantee that all considered elements would be controlled and thus facilitate the improvement of the quality process. Their adoption in the quality assurance approach will certainly be advantageous.
\end{abstract}

\section{KEYWORDS}

Quality Assessment, Agile Software Development, Scrum Metrics, Concept Map

\section{INTRODUCTION}

IT organizations aim to implement practices and processes in order to increase their product quality. Consequently, using metrics is so important to assess the quality within Agile Software Development (ASD). So, measuring quality through metrics guides managers and agile team to make decisions about projects in their organizations (Agarwal et al., 2014). To implement measurement culture in ASD, it is recommended not to collect several metrics, but only to keep the most pertinent ones. But, Scrum still now the most useful in software development organizations thanks to its fast response to change demands and also its short development cycle. It guarantees in short time to deliver a higher Business Value. In addition, Scrum is still used even for perform complex software development projects (Mishra and Abdalhamid, 2018).

Therefore, different approaches may be used in Scrum to select the appropriate metrics for quality measurement most of them are goal based approaches. As for example Goal Question Metric (GQM) which is a top-down approach that permits the identification of a desired goal, then a set of questions helps to achieve it and metrics helping to answer each question. Other approaches are based on stakeholders' goals (IT management, team members, Scrum Master and customers). But in spite of that, applying GQM is difficult in case of lacking stakeholders' vision of project goals since metrics selection is mostly conducted in a subjective way (Karklina and Pitra, 2018).On the other hand, Scrum is a framework process conducted and managed fundamentally around three components: Team, Artifacts and Events. We consider that this can be a perfect solution to categorize and select the appropriate Scrum metrics. In addition, Concept Mappings are graphical representations widely used to organize, understanding and share knowledge. They were originally developed by Novak for representing knowledge (Mejri et al., 2016). Inspired by this work, we decided adopting a concept map which conceptualizing metrics in Scrum process under its fundamental components. Metrics described by these concept maps were selected from literature. Moreover, according to the systematic mapping study conducted by (dos Santos et al., 2017); concept mapping was applied as an educational tool in multiple domains such as Computer Science. One from the main purposes of implementing CMs is knowledge organization. 
More precisely, via this work we proposed a solution to determine How to assess quality in Scrum process through metrics?

The remainder of the paper is structured as follows: in section 2, we introduce the background to discuss for Scrum method, Scrum metrics and concept map; in section 3, we describe; in section 3 we present the research works that have treated the similar questions; in section 4 we describe our proposed Concept map; finally, in section 5 we present conclusions and future works.

\section{BACKGROUND}

\subsection{Scrum}

Scrum is a framework performed on a sequence of Sprints (iterations). So, every Sprint is a time-box of one month or less which ends by creating a potentially releasable product Increment (Scrum Guides, 2017). Requirements are expressed in form of a simple description named "User Stories" (US) (Vannieuwenhuyze, 2013). The three fundamental Scrum' components are cited below.

Artifacts are the Product Backlog (PB) regroups all the US prioritized based on their Business Value by the Product Owner. The PB is dynamic; it is continually adapted in order to deliver a useful product satisfied client needs. Second, the Sprint Backlog regroups US selected from the PB with higher value of priority selected for the Sprint by the development team. It includes needing tasks to deliver it into "Done" Increment (Scrum Guides, 2017) (Vannieuwenhuyze, 2013).

Scrum team consists of three roles; a Scrum Master, a Product Owner and the development team described below (Scrum Guides, 2017) (Vannieuwenhuyze, 2013). They have all the necessary competences to accomplish the work. Scrum Master (SM) is responsible for promoting Scrum as defined in the Scrum guide. SM helps every member to understand Scrum theory, values, practices and rules. Product Owner (PO) is the unique responsible for managing the $\mathrm{PB}$, including its content, availability and order. $\mathrm{He} / \mathrm{sh}$ is also the spokesperson of future users and all stakeholders. Development Team is a group of professionals who develop US in the SB to deliver a potentially releasable increment at the end of each Sprint. The optimal development team size is between 3 to 9 persons, SM and PO aren't taken into account. Development team is self-organizing. Self-organizing means that only its members choose how to accomplish its work.

The Scrum events are Sprint planning Meeting, Daily Scrum, Sprint Review and Sprint Retrospective (Scrum Guides, 2017) (Vannieuwenhuyze, 2013). During the Sprint Planning Meeting, all Scrum team collaborates to select US from PB that will be developed in next Sprint. Selected US are estimate by development team through effort point. Once the Sprint begins, Daily Scrum is held every day and it lasts 15 minutes. During this meeting, development team updates columns of Sprint Backlog Board (to do, in progress, done, impediment). Once the Sprint ends, Sprint Review is held to inspect the increment and adapt the PB if required. Scrum team and key Stakeholders collaborate on work done in the Sprint and validate it. Then, the Sprint Retrospective is held. During this meeting, Scrum team inspects its work and constructs a plan of improvements which will be implemented in the next Sprint.

\subsection{Scrum Metrics}

As mentioned by Lord Kervin about the usage of metrics that it cannot be improved without measure it. Also Pfleeger affirms that metrics help to understand, monitor and improve done work and how it was done. In addition, Jones affirms that the most performant IT organizations such as Microsoft and IBM widely use metrics in their activities. (Kupiainen et al., 2015) proved that agile teams use it for many purposes focusing on different areas as for example sprint planning, detecting software process problems, tracking progress, etc. In ASD, the whole process and also Sprints must be planned and monitored.

So, measuring quality through metrics helps to identify and fixe process problems. In the case of Scrum plenty of metrics are adopted a base for quality assessment and improvement such as story point determined, $\%$ of automated test, test coverage, \% of US done in a sprint, etc. (dos Santos et al., 2017). 


\subsection{Concept Map}

Concept maps originally were developed in the courses of Novak's research program in 1972 at Cornell University as a result of following and understanding changes in children's knowledge of science (Novak and Canas, 2007). In fact, a concept map is a top-down diagram underlying relationships between concepts. It permits to show the systematic relationships between sub-concepts relating to a main concept (Eppler, 2006). Concept Maps represent organized knowledge which are needed to answer a focus question(s) (context dependent).

Thus, organized knowledge is composed of concepts and propositions. Concepts are perceived regularities or patterns, labelled with words and symbols and connected using linking words used to form propositions. Propositions are units of meaning and may be crosslinks showing interrelationships between map segments (Novak and Canas, 2006). Graphically, concepts are represented as boxes or bubbles connected with labelled connector arrows. The characteristic of concept maps is that concepts are hierarchically structured with the most inclusive concepts at the top of the map and the most specific concepts positioned at the bottom (Novak and Canas, 2006).

\section{STATE OF THE ART}

In literature, authors proposed different solutions in different manners to choose quality metrics in agile or Scrum software development process as stated below.

(Mahnic and Zabkar, 2008) proposed a Scrum measurement repository in order to collect project data and store measurement results. It results from a Scrum meta-model using entity-Relationship notation extended by adding appropriate metrics. These measures have been chosen referring to Scrum and Measurement Analysis Process Area of CMMI practices. So, proposed measures aim to monitor satisfaction of Scrum stakeholders which refers to process performance. (Mahnic and Vrana, 2007) presented a metrics plan which monitors and improves continuously the software development process performance in Scrum method. This plan consists of a set of indicators and corresponding metrics that measure the performance based on stakeholders' goals (IT management, Team members, and customers). In addition, each indicator can be evaluated using direct metrics which are calculated by an appropriate project management tool. So their proposed stakeholder-driven Scrum process performance measurement provides a guarantee for process and software quality. An agile metric mind-map has been mentioned by (Oza and Korkala, 2012) which regroups the most useful agile metrics. This mind-map classifies agile metrics measuring quality into seven categories: engineer, strategic, economic, test, iteration, code, automation and project management.

(Downey and Sutherland, 2013) proposed a set of nine fundamental Scrum metrics measure and monitor team performance if problems arise; Velocity, Work Capacity, Focus Factor, Percentage of Adopted Work, Percentage of Found Work, Accuracy of Estimation, Accuracy of Forecast, Targeted Value Increase, Success at Scale, and the Win/Loss Record of the Team. By this way, Scrum team maintains a high energy state that generates a hyper-productivity. We consider that collecting these quality data on team performance is one of the powerful information allowing to deliver a software of high quality. (Karklina and Pitra, 2018) proposed an approach to identify quality metrics and retrieve data from project management systems. This approach was represented as a process workflow. It permits defining the appropriate metrics for the proposed goals using GQM approach and choosing a project management tool (JIRA, Redmine, etc.) according to required features. As a result, authors proved that this approach can be used successfully for quality control in agile projects and helps stakeholders in their decision making. (Boerman et al., 2015) presented a measuring and reporting model for quality, progress and predictions in agile projects. To design this model, metrics have been derived using GQM approach. As a result, the measurement model can be considered as a way which describes these metrics. We conclude that indicators proposed at this work on quality are helpful especially for project owners (external stakeholders) by monitoring quality and controlling project status only via PB. Another solution was also intended to deal with quality requirements. (Guzmán et al, 2017) proposed Q-Rapids methodology to manage quality and functional requirements concurrently in Rapid Software Development. Software quality is then based on quality-related metrics by adopting Quamoco approach to define quality model. Q-Rapids helps decision makers to identify and monitor quality problems continuously throughout the development process. 
All these work have used metrics in order to measure, monitor and improve software/process quality. These metrics are selected based on different approaches. In contrast, our contribution in this work is based on a concept map as a different approach for choosing appropriate metrics for Scrum development process.

\section{PROPOSED CONCEPT MAPS}

As specified by (Novak and Canas, 2006) and (Novak and Canas, 2007), to construct a good concept map, we followed the following steps: First, define the domain of knowledge by setting an appropriate focus question which will be answered by the mapped knowledge. In our case, the focus question is "How to assess quality in Scrum process through metrics?". Second, identify key concepts: concepts defined in our map are presented in next sub-sections. Scrum components are all represented (PB, SB, Sprint Review, etc.) as concepts and their corresponding metrics too. Third, construct a preliminary concept map: accordingly, we used CmapTools as software tool. Forth and finally, revise the concept map: readjusting our preliminary concept map based on experts opinion. For a more comprehensive and readable concept map, we proceeded from general to more specific concepts. Thus, at the first stage, we constructed a "macro concept map" classifying metrics in Scrum process according to belongs to its 3 principal aspects which are team, events (ceremonies) and artifacts. Then, "micro concept maps" are constructed to show metrics related to each specific concept of this macro map. So, these maps are described below.

\subsection{Macro Concept Map}

As illustrated in figure 1, Scrum process metrics can be categorized according to Events, Team and Artifacts. Events derived metrics correspond to metrics measured during a given event. They include Sprint Planning meeting derived metrics, Daily Scrum derived metrics, Sprint Review derived metrics and Sprint Retrospective derived metrics. Therefore, this map describes when metrics can be derived. Team related metrics include Team Performance.

Moreover, Size of team and Percentage of team member's engagement are metrics that refer to teams and calculated during the Sprint Planning Meeting. Other metrics are related to artifacts which include PB and SB. They are consequently composed of PB's User Story and SB's User Story related metrics.

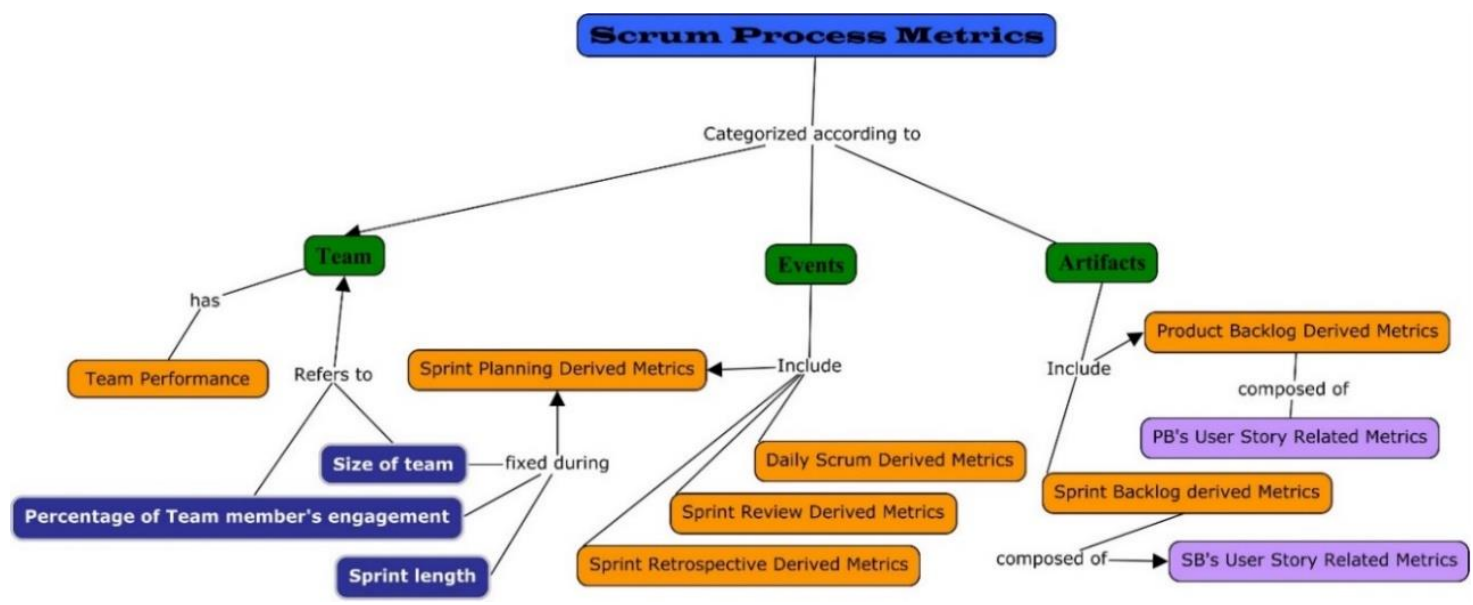

Figure 1. Macro map for Scrum process metrics

\subsection{Micro Concept Maps}

Figure 2 shows a micro map for Team Performance which is measured through a set of metrics as for example Velocity and work capacity. 


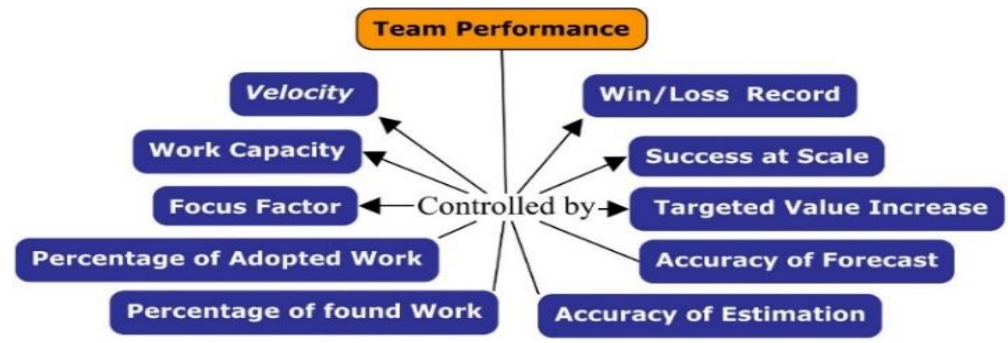

Figure 1. Micro map for team performance

Figure 3 shows a micro map for expanding more details about the following concepts: Daily Scrum, Sprint Review and Sprint Retrospective related metrics. Metrics determined during Daily Scrum are number of impediments for a given task, number of tasks in progress, number of tasks realized/done and number of tasks to do. Metrics determined during Sprint Review are total number of errors, total number of accepted US and total number of rejected US. Metrics determined during Sprint Retrospective and calculated for a given Sprint are total number of tasks in Sprint, number of tasks completed in Sprint, etc.

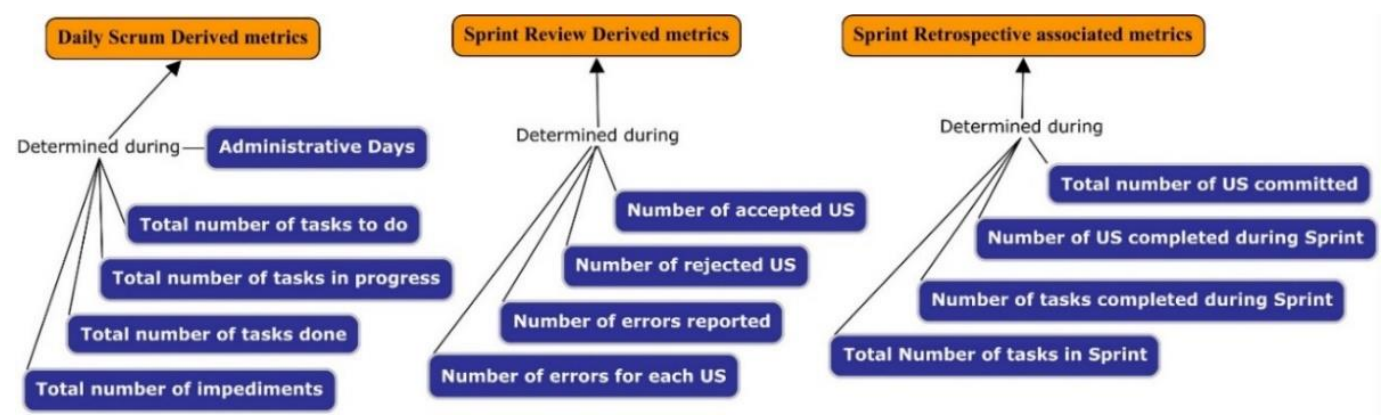

Figure 2. Micro map for metrics determined during events

In addition, Figure 4 shows a micro map for expanding more details about the following concepts: SB's User Story and PB's User Story. The corresponding metrics are determined for each of these concepts. SB's User Story related metrics are number of unit tests, number of Acceptance tests, number of functional tests, etc. These metrics are themselves categorized into two main classes: test related metrics and task related metrics. PB's User Story related metrics are total number of US, total number of deleted US, etc.

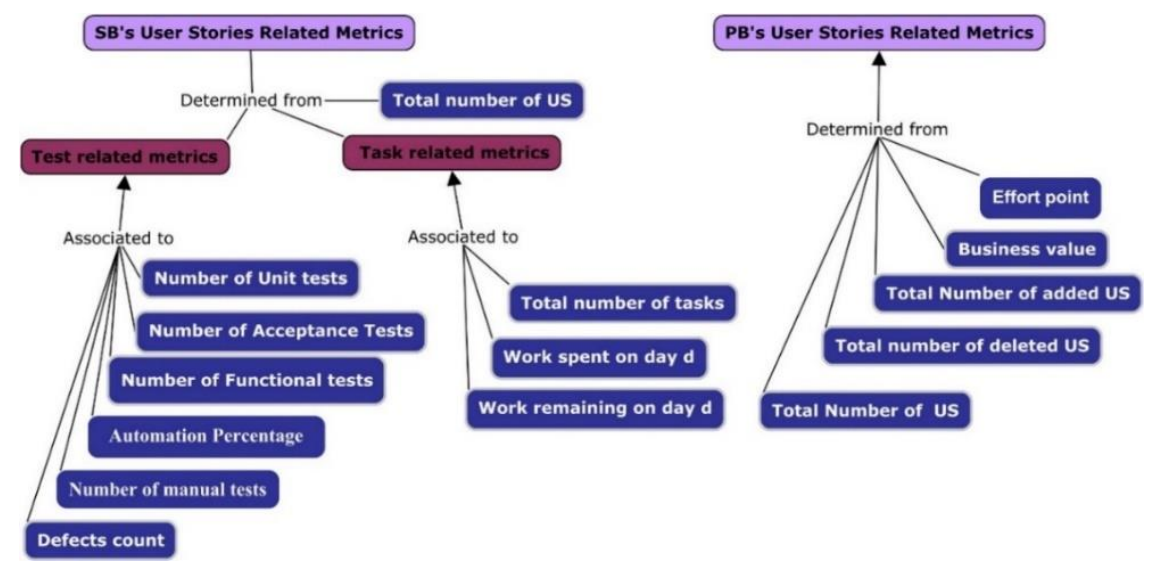

Figure 3. Micro map for metrics determined from Sprint Backlog and Product Backlog 


\section{CONCLUSION AND FUTURE WORK}

In this work, we proposed a conceptualization of Scrum software process metrics using concept maps. As a result, these concept maps classify Scrum metrics firstly according to Scrum fundamental elements of (team, events and artifacts) as concepts in a macro map. Then, each of these concepts is more detailed (presenting appropriate metrics) in micro maps. To conclude, our contribution is a new means to select appropriate metrics based on this classification that promotes quality assessment to improve the quality process and consequently quality product. These concept maps are a graphical representation dedicated to Scrum metrics and their classifications according to the three fundamental elements of Scrum. They offer a means for deeper understanding knowledge related to their usefulness and a guarantee that the basic elements are controlled.

In terms of future work, it would be interesting to accomplish the fourth step of the construction of the concept map (mentioned in section 4) to validate incorporated metrics from practitioners and Scrum experts. Additionally, we aim to implement, in an agile way, these concept maps in Scum process in order to facilitate Scrum Master' decision-making through the visualization of their metrics measurement results in a dashboard. Besides, determining when and how to collect data for measuring these metrics without distracting Scrum teams would also be a challenge.

\section{REFERENCES}

Agarwal, A., Garg, N.K and Jain, A., 2014. Quality Assurance for Product Development using Agile. International Conference $\quad$ on Reliability, Optimization and Information Technology. Faridabad, India, pp. 44-47.

Boerman, MP. et al, 2015. Measuring and Monitoring Agile Development Status. Proceedings of the Sixth International Workshop on Emerging Trends in Software Metric. Florence, Italy, pp. 54-62.

dos Santos, V. et al., 2017. Analyzing the Use of Concept Maps in Computer Science: A Systematic Mapping Study. In Informatics in Education, Vol. 16, No. 2, pp. 257-288.

Downey, S. and Sutherland, J., 2013. Scrum Metrics for Hyperproductive Teams: How They Fly like Fighter Aircraft. 46th Hawaii International Conference on System Sciences. Wailea, Maui, HI, USA.

Eppler, M., 2006. A comparison between concept maps, mind maps, conceptual diagrams, and visual metaphors as complementary tools for knowledge construction and sharing. In Information Visualization, Vol. 5, No. 3, pp. 202-210.

Guzmán, L. et al, 2017. How Can Quality Awareness Support Rapid Software Development?. 23d International Conference on Requirements Engineering: Foundation for Software Quality. Essen, Germany, pp 167-173.

Karklina, K. and Pitra, R., 2018. Quality Metrics in Agile Software Development Projects. In Information Technology and Management Science, vol. 21, pp. 54-59.

Kupiainen, E., Mäntylä, M.V., Itkonen, J., 2015. Using metrics in Agile and Lean Software Development - A systematic literature review of industrial studies. In Information and Software Technology, Vol. 62, pp. 143-163.

Mahnic, V. and Vrana, I., 2007. Using stakeholder-driven process performance measurement for monitoring the performance of a Scrum-based software development process. In Electrotechnical Review: Ljubljana, Slovenija, Vol. 75, No. 5, pp. 241-247.

Mahnic V. and Zabkar, N., 2008. Measurement repository for Scrum-based software development process. CEA'08 Proceedings of the 2nd WSEAS International Conference on Computer Engineering and Applications. Acapulco, Mexico, pp. 23-28.

Mejri, A. et al., 2016. Representing Business Process Flexibility using Concept Maps. Conference on ENTERprise Information Systems / International Conference on Project MANagement / Conference on Health and Social Care Information Systems and Technologies. Porto, Portugal.

Mishra, D. and Abdalhamid, S., 2018. Software Quality Issues in SCRUM: A Systematic Mapping. In Journal of Universal Computer Science, vol. 24, no. 12, pp. 1690-1616.

Novak, JD. and Canas, AJ., 2007. Theoretical origins of concept maps, how to construct them, and uses in education. In Reflecting Education, Vol. 3, No. 1, pp. 29-42.

Novak, JD. and Gowin, DB., 1982. Learning how to learn. In Cambridge University Press.

Oza, N. and Korkala, M., 2012. Lessons Learned In Implementing Agile Software Development Metrics. UK Academy for Information Systems Conference Proceedings 2012. UK, United Kingdom, pp 38-50.

Scrum Guides: The Definitive Guide to Scrum: The Rules of the Game (2017), available at https://www.scrumguides.org/docs/scrumguide/v2017/2017-Scrum-Guide-US.pdf 